\title{
Redesigning the Door Reduces the Potential for Finger Injuries in Children
}

\author{
Ivan Yang ${ }^{1}$, Loren Yamamoto ${ }^{2, *}$ \\ ${ }^{1}$ Department of Biology, University of Hawaii, Honolulu, United States \\ ${ }^{2}$ Department Pediatrics, University of Hawaii, Honolulu, United States
}

Email address:

ivanyang@hawaii.edu (I. Yang), loreny@hawaii.edu (L. Yamamoto)

${ }^{*}$ Corresponding author

\section{To cite this article:}

Ivan Yang, Loren Yamamoto. Redesigning the Door Reduces the Potential for Finger Injuries in Children. American Journal of Pediatrics. Vol. 7, No. 1, 2021, pp. 34-38. doi: 10.11648/j.ajp.20210701.18

Received: February 27, 2021; Accepted: March 17, 2021; Published: March 30, 2021

\begin{abstract}
The standard door is a safety hazard for young children often resulting in crush injuries, nail avulsions, fractures, lacerations, and amputations. This type of injury occurs mostly in households and may sometimes happen to adults, although this is less common. The simplicity of the door's design has continually become a concern mostly due to its potential to cause injury. The purpose of this study is to redesign the door to reduce the risk of finger injuries in children. A safety door was designed and a model of it was constructed for study. Its two major features utilize silicone rubber fins at the pinch points and a "piano" hinge running the entire door length (instead of the conventional two or three separate hinges) to eliminate the space between the hinges. Ten door closures using five finger models (beef stick, green beans, string cheese, crayons, red licorice twists) were performed to observe and assess the resulting injuries from the safety door compared to a standard door. The severity of the resulting injuries was scored using a $0-8$ numerical score. The collected data were recorded in a computer spreadsheet for statistical analysis. The safety door resulted in 1 amputation ( $0.7 \%)$ compared to 120 amputations (out of 200 door closures) in the standard door $(60 \%$, $\mathrm{p}<0.0001)$ and had significantly lower numerical injury scores $(0.2 \mathrm{vs} 4.8, \mathrm{p}=0.0001)$. The safety door is less injurious than the conventional door and re-designing the door will likely lower the risk of finger injuries in young children.
\end{abstract}

Keywords: Door Injuries, Finger Injuries, Injury Prevention, Door Re-design

\section{Introduction}

Door-related finger injuries are common [1-6]. Essentially, doors are dangerous for the fingers of young children [7]. The resulting injuries are often crush injuries, nail avulsions, fractures, lacerations, and amputations leading to pain, reduction of function, shortening of the phalanx, and distress for both the child and parent [1-3, 8-13]. The door is a classic old design and we believe that it can be improved to reduce its guillotine-like injury potential while maintaining its security and weather protection properties. The purpose of this study is to propose a door design with a reduced risk for significant finger injuries and to test it in a model.

\section{Method}

A safety door model was constructed using various wood materials and soft silicone rubber fins (thickness $1.1 \mathrm{~mm}$ ) following the schematic layout in Figure 1. The silicone fins were cut by hand to a length of $5 \mathrm{~cm}$, assembled into a module, and inserted into the door. The silicone fins module was layered by hand with an air gap space between each silicone fin to provide an insulating and weatherproofing feature of the door while being flexible and non-injurious to the finger. Figure 2 shows this safety feature on the doorknob side of the door. A similar module was also inserted into the hinge side of the door. The remainder of the model's door was a standard wood interior (hollow) door and frame used as a control.

To test the injury potential of the door prototype, we used models to mimic children's fingers (ages 3 to 10) which has an average diameter of $1.3 \mathrm{~cm}(0.8-1.9 \mathrm{~cm})$ for the ring finger [4]. In one study, the most commonly injured finger was the ring finger in children under 5 years of age [3], thus, the diameter of the models we used closely mimic this. Models included beef sticks (Pup-Peroni dog treat sticks, J. M. Smucker Company, 
Orrville, $\mathrm{OH}$ ) (average diameter $1.2 \mathrm{~cm}$ ), tubular string cheese (cut longitudinally in fourths resulting in an approximate diameter of $0.8 \mathrm{~cm}$ ), crayons (approximate diameter $0.8 \mathrm{~cm}$ ), fresh (not frozen or canned) green beans (Green Giant, Parsippany, NJ) (approximate diameter $0.9 \mathrm{~cm}$ ), and red licorice twists (Red Vines, American Licorice Company, La Porte, IN) (average diameter $0.7 \mathrm{~cm}$ ). The objects were then placed at the injury position at approximately 22 degrees (half of a 45 degree angle) measured from the door frame, in the control door and the safety door on both the door knob side and the hinge side of the door, approaching from the inside of the door and the outside of the door (four possible combinations). The door was closed and the result of the closure was assessed and scored as: amputated (score $=5$ ), crushed (reduction of original diameter) and angulated (score $=4$ ), obviously visible crush $($ score $=3$ ), barely visible crush (score $=2$ ), no crush but lacerations visible ( score $=1$ ), or visibly uninjured (score $=0$ ). In some instances, the object was crushed and amputated. This would be clearly more difficult to repair and would result in a more severe deformity than amputation alone, so a greater severity score was achieved when the two elements were combined as in: amputated and crushed (score $=8$ ) and amputated with barely visible crush (score=7). Each item was repeated 10 times for each door type and location. Figure 3 shows an example using red licorice twists closing in the silicone fins safety portion of the door compared to the conventional part of the door.

\section{Results}

Amputation rates are summarized in Table 1. In aggregate there were significantly fewer amputations with the safety door compared to the standard door $(\mathrm{p}<0.0001)$.

Injury scores in the two door types are summarized in Table 2, which displays the mean scores, standard deviations (SD), and the $95 \%$ confidence interval of the mean (95\% CIM). This demonstrates that in all comparisons with all the models used, the safety door had significantly lower injury severity scores than the standard door.

Table 1. Amputations in each of the doors using various models (10 trials per model item). *Not applicable because the safety door's piano hinge eliminates this possible injury.

\begin{tabular}{|c|c|c|}
\hline & Safety Door Amputations & Standard Door Amputations \\
\hline \multicolumn{3}{|l|}{ Hinge side of door } \\
\hline \multicolumn{3}{|l|}{ Inside to out } \\
\hline Beef stick & $\mathrm{N} / \mathrm{A}^{*}$ & $10 / 10$ \\
\hline Green bean & N/A* & $0 / 10$ \\
\hline Red vines & N/A* & $0 / 10$ \\
\hline Crayon & $\mathrm{N} / \mathrm{A}^{*}$ & $10 / 10$ \\
\hline String cheese & N/A* & $0 / 10$ \\
\hline All & $\mathrm{N} / \mathrm{A}^{*}$ & $20 / 50$ \\
\hline \multicolumn{3}{|l|}{ Outside to in } \\
\hline Beef stick & $0 / 10$ & $10 / 10$ \\
\hline Green bean & $0 / 10$ & $0 / 10$ \\
\hline Red vines & $0 / 10$ & $0 / 10$ \\
\hline Crayon & $0 / 10$ & $10 / 10$ \\
\hline String cheese & $0 / 10$ & $0 / 10$ \\
\hline All & $0 / 50$ & $20 / 50$ \\
\hline All hinge side & $0 / 50$ & $40 / 100$ \\
\hline \multicolumn{3}{|l|}{ Doorknob side of door } \\
\hline \multicolumn{3}{|l|}{ Inside to out } \\
\hline Beef stick & $1 / 10$ & $10 / 10$ \\
\hline Green bean & $0 / 10$ & $10 / 10$ \\
\hline Red vines & $0 / 10$ & $0 / 10$ \\
\hline Crayon & $0 / 10$ & $10 / 10$ \\
\hline String cheese & $0 / 10$ & $10 / 10$ \\
\hline All & $1 / 50$ & $40 / 50$ \\
\hline \multicolumn{3}{|l|}{ Outside to in } \\
\hline Beef stick & $0 / 10$ & $10 / 10$ \\
\hline Green bean & $0 / 10$ & $10 / 10$ \\
\hline Red vines & $0 / 10$ & $0 / 10$ \\
\hline Crayon & $0 / 10$ & $10 / 10$ \\
\hline String cheese & $0 / 50$ & $10 / 10$ \\
\hline All & $0 / 50$ & $40 / 50$ \\
\hline All doorknob side & $1 / 100$ & $80 / 100$ \\
\hline All both sides of door & $1 / 150$ & $120 / 200$ \\
\hline
\end{tabular}


Table 2. Mean injury scores (SD) and 95\%CIM in each of the doors using various models. ${ }^{*}$ Not applicable because the safety door's piano hinge eliminates this possible injury.

\begin{tabular}{|c|c|c|c|c|}
\hline & \multicolumn{2}{|l|}{ Safety Door } & \multicolumn{2}{|c|}{ Standard door } \\
\hline & Mean & 95\%CIM & Mean & 95\%CIM \\
\hline \multicolumn{5}{|l|}{ Hinge side of door } \\
\hline Beef stick & N/A* & & $8(0)$ & 8 to 8 \\
\hline Green bean & N/A* & & $3(0)$ & 3 to 3 \\
\hline Red vines & N/A* & & $2.8(0.4)$ & 2.5 to 3.1 \\
\hline Crayon & N/A* & & $8(0)$ & 8 to 8 \\
\hline String cheese & $\mathrm{N} / \mathrm{A}^{*}$ & & $3(0)$ & 3 to 3 \\
\hline All & N/A* & & $5(2.5)$ & 4.3 to 5.7 \\
\hline \multicolumn{5}{|l|}{ Outside to in } \\
\hline Beef stick & $0.4(1.3)$ & -0.4 to 1.2 & $8(0)$ & 8 to 8 \\
\hline Green bean & $0(0)$ & 0 to 0 & $3(0)$ & 3 to 3 \\
\hline Red vines & $0(0)$ & 0 to 0 & $2.9(0.3)$ & 2.7 to 3.1 \\
\hline Crayon & $0(0)$ & 0 to 0 & $8(0)$ & 8 to 8 \\
\hline String cheese & $0.3(0.5)$ & 0.0 to 0.6 & $3(0)$ & 3 to 3 \\
\hline All & $0.1(0.6)$ & -0.03 to 0.3 & $5(2.5)$ & 4.3 to 5.7 \\
\hline All hinge side & $0.1(0.6)$ & -0.03 to 0.3 & $5(2.5)$ & 4.5 to 5.5 \\
\hline \multicolumn{5}{|l|}{$\begin{array}{l}\text { Doorknob side of door } \\
\text { Inside to out }\end{array}$} \\
\hline Beef stick & $0.5(1.6)$ & -0.5 to 1.5 & $5(0)$ & 5 to 5 \\
\hline Green bean & $0(0)$ & 0 to 0 & $5(0)$ & 5 to 5 \\
\hline Red vines & $0(0)$ & 0 to 0 & $3.1(0.3)$ & 2.9 to 3.3 \\
\hline Crayon & $0(0)$ & 0 to 0 & $5(0)$ & 5 to 5 \\
\hline String cheese & $0.1(0.3)$ & -0.1 to 0.3 & $5(0)$ & 5 to 5 \\
\hline All & $0.1(0.7)$ & -0.1 to 0.3 & $4.6(0.8)$ & 4.4 to 4.8 \\
\hline \multicolumn{5}{|l|}{ Outside to in } \\
\hline Beef stick & $0(0)$ & 0 to 0 & $5(0)$ & 5 to 5 \\
\hline Green bean & $0(0)$ & 0 to 0 & $5(0)$ & 5 to 5 \\
\hline Red vines & $0(0)$ & 0 to 0 & $3(0)$ & 3 to 5 \\
\hline Crayon & $0(0)$ & 0 to 0 & $5(0)$ & 5 to 5 \\
\hline String cheese & $1(0)$ & 1 to 1 & $5(0)$ & 5 to 5 \\
\hline All & $0.2(0.4)$ & 0.1 to 0.3 & $4.6(0.8)$ & 4.4 to 4.8 \\
\hline All doorknob side & $0.2(0.6)$ & 0.05 to 0.3 & $4.6(0.6)$ & 4.5 to 4.8 \\
\hline All both sides of door & $0.2(0.6)$ & 0.1 to 0.2 & $4.8(1.9)$ & 4.5 to 5 \\
\hline
\end{tabular}

\section{Discussion}

Finger injuries in children clearly occur with doors [1-3, 5-7]. If the door was a recent product brought to the market, it might not pass consumer safety expectations because of the numerous reports of its injury potential.

Our door design has demonstrated injury reduction benefits in several models. It would not be possible to use real fingers, thus, a variety of models were used instead, to assess its injury reduction potential.

Our door was designed for injury reduction, weather proofing, and security. This study tested one injury reduction portion of the door, namely the part where the door closes onto the door frame by creating a soft and flexible closure. Another safety that is incorporated into our safety door is to use a "piano hinge" instead of the standard two or three door hinges that suspend the door in the door frame. The standard hinges have gaps between them that permit a child's finger to enter from the inside or outside of the door (see figure 4). A "piano hinge" is a single long hinge that has no gaps, thus a child's finger cannot enter the door gap from the inside, but this doesn't prevent an injury from the outside (see figure 5). This is called a "piano hinge" because it is the type of hinge used for the lid that covers the piano keys or the strings of a grand piano.

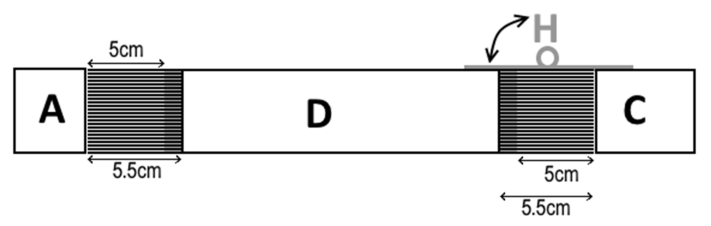

Figure 1. Top schematic layout of door prototype that includes door frame (A $\& C)$, main door (D), door hinge $(H)$ which is a piano hinge, and silicone plate (grey lines between $A \& D$ and $D \& C$ ).

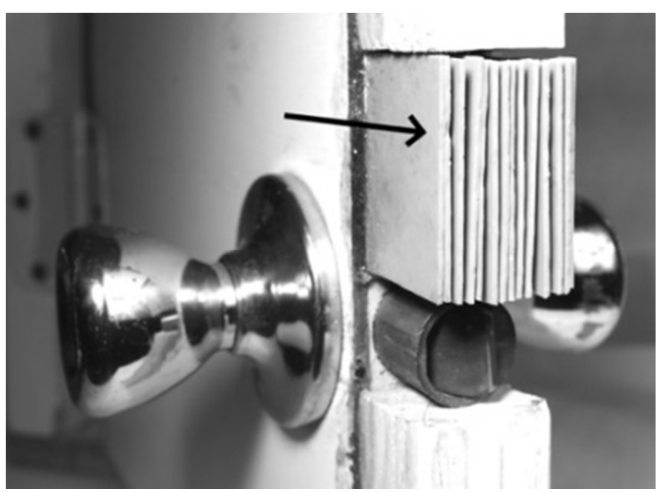

Figure 2. The hand cut silicone fins (arrow) section insert on the test door models the injury potential of this safety door feature. It appears irregular because it is made by hand. Each fin is $1.5 \mathrm{~mm}$ thick with an intended space of $1.1 \mathrm{~mm}$ between each fin. 


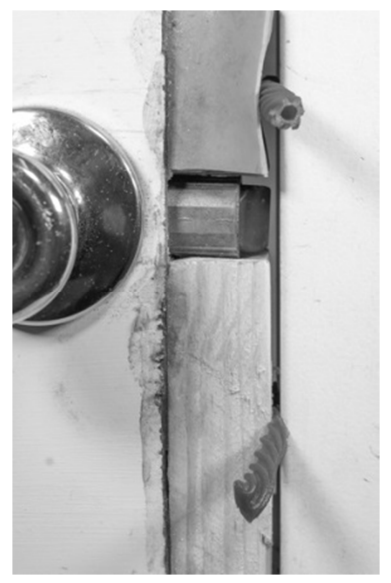

Figure 3. The tubular structures are red licorice twists. As the door has closed, the safety silicone fins in the upper door have closed on the upper red licorice twists leaving it intact, while the lower conventional door frame has closed on the lower red licorice twists which has crushed and amputated it.

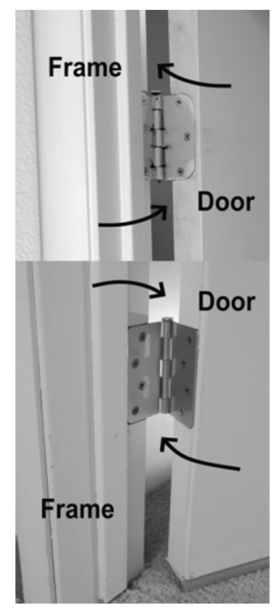

Figure 4. Two different conventional door hinges. These hinges create gaps between the door and the door frame. Fingers can enter this gap (arrows) either above or below the door hinge from either the outside or the inside, resulting in a potential for finger injury if the door is closed.

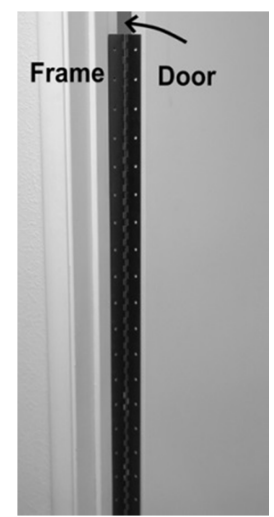

Figure 5. This shows a "piano hinge" holding the door to the door frame. For comparison purposes only, the gap above the piano hinge (arrow) permits a finger to enter. But along the length of the piano hinge which could run the entire length of the door, there are no gaps between the door and the door frame, eliminating any risk of injury from the inside of the door. The potential for finger injury from the outside of the door remains, but the length of the finger would be limited since it would be stopped by the piano hinge. For example, the crush point could be at the distal interphalangeal joint, but less likely to reach the proximal interphalangeal joint.
Weather proofing. The silicone fins also provide a reasonable seal that prevents wind, rain, heat, and bugs from entering through the door. This additional feature allows us to preserve the original weather proofing qualities of the conventional door. It might actually be better than a rigid conventional door which can permit air leaks and small entry points, compared to the flexible silicone fins that can more effectively reduce air leaks $[14,15]$.

Security elements of the door must be preserved for the door to be functional. Because the silicone fins are soft, they appear to be easy to cut with a knife or scissors resulting in less security; however, this is not true because the fins are mobile, fairly strong and difficult to cut, and there are multiple layers, such that they can only be cut with significant difficulty [15]. Even if the fins are cut, an intruder would not be able to enter with this alone. Gaining entry by an intruder would still be identical to that of a standard door. Defeating the security of standard door can be achieved with moderate or extreme measures. To gain entry with moderate measures, an intruder must still either cut the door closure bolt, the hinges, or create a larger gap or hole that permits a hand or device to open the door from the inside. To gain entry with extreme measures, a ramming device would defeat any conventional door. Substituting the original hinge with a "piano" hinge will further strengthen its security elements because it is almost impossible to remove the hinge pin and cutting the hinge is more difficult since it requires a cut along the entire length of the hinge.

\section{Conclusion}

Our safety door is less injurious than the conventional door demonstrating that re-design considerations can reduce the likelihood of door finger injuries in children. The modifications implemented on the safety door also allows for an enhanced safety feature along with the retention of its weatherproofing properties.

\section{References}

[1] Doraiswamy NV. (1999). Childhood finger injuries and safeguards. Inj Prev 1999; 5: 298-300. doi: 10.1136/ip.5.4.298.

[2] King PA. Trapped Finger Injury. Med J Australia 1979; 2 (11): 580-582. doi: 10.5694/j.1326-5377.1979.tb127193.x.

[3] Liu WH, Lok J, Lau MS, et al. Mechanism and epidemiology of paediatric finger injuries at Prince Wales Hospital in Hong Kong. Hong Kong Med J 2015; 21 (3): 237-242.

[4] Hohendorff B, Weidermann C, Burkhart KJ, et al. Lengths, girths, and diameters of children's fingers from 3 to 10 years of age. Ann Anat - Anatomischer Anzieger, 2010: 192 (3): 156-161. doi: 10.1016/j.aanat.2010.03.002.

[5] Smith, G. (2012). Children Treated in United States Emergency Departments for Door-Related Injuries, 1999-2008. Clinical Pediatrics., $\quad 51 \quad$ (3), 226-232. https://doi.org/10.1177/0009922811423308. 
[6] Venkatesh, A., Khajuria, A., \& Greig, A. (2020). Management of pediatric distal fingertip injuries: a systematic literature review. Plastic and Reconstructive Surgery Global Open, 8 (1).

[7] Routley, V., \& Valuri, J. (1993). Home injuries.

[8] Satku, M., Puhaindran, M. E., \& Chong, A. K. S. (2015). Characteristics of fingertip injuries in children in Singapore. Hand surgery, 20 (03), 410-414.

[9] Fetter-Zarzeka, A., \& Joseph, M. M. (2002). Hand and fingertip injuries in children. Pediatric Emergency Care, 18 (5), 341-345.

[10] Weir, Y. (2021). Fingertip injuries in children: a review of the literature. Emergency nurse, 29 (2).

[11] McGhee, S., Gonzales, J., Nadeau, C., \& Ortega, J. (2021). Mallet Finger Injuries: the signs, symptoms, diagnosis and management. Emergency Nurse, 29 (2).
[12] Reid, D. B., Shah, K. N., Eltorai, A. E., Got, C. C., \& Daniels, A. H. (2019). Epidemiology of finger amputations in the united states from 1997 to 2016. Journal of Hand Surgery Global Online, 1 (2), 45-51.

[13] Haehnel, O., Plancq, M. C., Deroussen, F., Salon, A., Gouron, R., \& Klein, C. (2019). Long-term outcomes of Atasoy flap in children with distal finger trauma. The Journal of hand surgery, 44 (12), 1097-e1.

[14] Hou, S. S., \& Kuo, P. L. (1999). Function and performance of silicone copolymers, 3. Synthesis and properties of a novel siliconized acrylic monomer containing three reactive sites. Macromolecular Chemistry and Physics, 200 (11), 2501-2507.

[15] Xu, J., Razeeb, K. M., \& Roy, S. (2008). Thermal properties of single walled carbon nanotube-silicone nanocomposites. Journal of Polymer Science Part B: Polymer Physics, 46 (17), 1845-1852. 